\title{
18. On the Red Carbon Stars
}

\author{
By Yoshio Fujita \\ Department of Astronomy, University of Tokyo \\ (Comm. by Y. Hagihara, M.J.A., Feb. 18, 1955)
}

The theoretical consideration of the spectral sequence of the late type stars has been taken from the dissociative equilibrium of molecules and it was concluded that the existence of the branches in the late type stars might be explained by the continuous change in the relative abundance of the elements $\mathrm{O}, \mathrm{N}$, and C. ${ }^{1)}$ Further, there is some possibility to suggest that we might also classify the late type stars into three branches, in each of which either oxygen, carbon or nitrogen may act in some degree the dominant role. ${ }^{2)}$ As one of the main conclusions, it was pointed out that the S-type stars could be considered as cases of intermediate carbon abundance, with the role of nitrogen rather undefined. One of the interesting features which should appear in the stellar spectra is that the increased carbon abundance weakens the strength of the TiO bands and thus the opacity due to carbon compounds becomes dominant. Bidelman $^{32}$ observed carbon stars with greatly varying strengths of the $\mathrm{C}_{2}$ bands and also S-type stars with greatly varying strengths of the $\mathrm{ZrO}$ bands. Thus it is quite certain that observational evidence points to a remarkable relation between the carbon and the S-type stars. . $^{4}$

An important point to which attention may be drawn in regard to the late type stars, above described, is that Keenan and Morgan ${ }^{5)}$ classified $\mathrm{R}$ and $\mathrm{N}$ type stars into a single type $\mathrm{C}$. Our paper aims. to try to make clear how C classification works as observed from the spectra of a medium dispersion.

The observed materials here investigated ${ }^{6)}$ are Coudé spectro-

1) Y. Fujita: Jap. Journ. Astr. Geophys., 13, 21 (1935); ibid.,17, 17 (1939); ibid., 18, 45, 177 (1940); Proc. Phys. Math. Soc., Japan, 20, 484 (1938).

2) Y. Fujita: Journ. Phys. Soc., Japan, 2, 204 (1947); Publ. Astr. Soc., Japan, 1, 23 (1949).

3) W. P. Bidelman: Comm. presented to the 5th International Colloquium of Astrophysics (Liège, 1953).

4) Y. Fujita: Ap. J., 113, 626 (1951); ibid., 119, 141 (1954); Publ. Astr. Soc., Japan, 4, 81 (1952); Trans. IAU, 8, 828 (1952).

P. C. Keenan: Ap. J., 120, 484 (1954).

5) P. C. Keenan and W. W. Morgan: Ap. J., 94, 501 (1941).

6) The preliminary report has been read at the 6th International Colloquium of Astrophysics in Liège last year and it is now in press. The detailed discussion with: the wave-length table will be published in a later paper. 
grams of five red carbon stars RY Dra, V Aql, U Hya, U Cyg, and WZ Cas obtained at the McDonald Observatory. The details are given in Table I.

Table I

\begin{tabular}{|c|c|c|c|c|c|c|c|}
\hline \multicolumn{2}{|c|}{ Star } & Plate No. & \multicolumn{2}{|l|}{ Date } & \multirow{2}{*}{$\frac{\text { Setting }}{7.0}$} & \multirow{2}{*}{$\begin{array}{c}\begin{array}{c}\text { Exposure } \\
\text { (minutes) }\end{array} \\
100\end{array}$} & \multirow{2}{*}{$\frac{\text { Emulsion }}{\mathrm{I}-\mathrm{N} *}$} \\
\hline $\mathrm{RY}$ & Dra & CIR 25 & April 26, & 1946 & & & \\
\hline $\mathrm{V}$ & Aql & , & ," & & 10.0 & 90 & \\
\hline $\mathrm{V}$ & $\mathrm{Aql}$ &, & , & & 8.5 & 90 & \\
\hline RY & Dra & CIR 26 & , & & 11.5 & 20 & $103 \mathrm{aF}$ \\
\hline $\mathrm{RY}$ & Dra &, &, & & 13.0 & 20 & \\
\hline $\mathrm{U}$ & Hya & CIR 28 & April 27, & 1946 & 9.5 & 30 & $\mathrm{I}-\mathrm{N} *$ \\
\hline $\mathrm{U}$ & Hya &, &, & & 8.5 & 30 & \\
\hline $\mathrm{U}$ & Hya &,, &, & & 7.0 & 30 & \\
\hline$R Y$ & Dra & , &, & & 8.5 & 100 & \\
\hline $\mathrm{RY}$ & Dra & CIR 31 & April 28, & 1946 & 10.0 & 100 & $\mathrm{I}-\mathrm{N} *$ \\
\hline $\mathrm{U}$ & Cyg & CIR 118 & May 20 , & 1946 & 9.25 & 50 & $\mathrm{I}-\mathrm{N} *$ \\
\hline WZ & Cas & , & , & & 9.5 & 60 & \\
\hline WZ & Cas & , &, & & 7.75 & 60 & \\
\hline $\mathrm{U}$ & Cyg & CIR 119 & , & & 11.0 & 20 & \\
\hline WZ & Cas & , &, & & 11.25 & 20 & \\
\hline
\end{tabular}

The spectral region investigated is shown in Table II.

Table II

\begin{tabular}{|c|c|c|c|}
\hline \multicolumn{2}{|c|}{ Star } & \multirow{2}{*}{$\begin{array}{c}\text { Spectral region } \\
5717 \AA-8670 \AA\end{array}$} & Dispersion \\
\hline RY & Dra & & \multirow{5}{*}{$49 \AA / \mathrm{mm}$} \\
\hline & Aql & $6451 \quad-8026$ & \\
\hline $\mathrm{U}$ & Hya & $\begin{array}{ll}6727 & -8712\end{array}$ & \\
\hline $\mathrm{U}$ & Cyg & $5961-7725$ & \\
\hline WZ & Cas & $5856-8400$ & \\
\hline
\end{tabular}

Microphotometric tracing of all these spectrograms has been carried out with the registering microphotometer of the Yerkes Observatory. The wave-lengths have been derived and corrected for the radial velocity corresponding to each star. As no special consideration has been given for the spectral photometry, only the visual estimation of the intensity of each line has been done. The identification of each line was made with the help of MIT Table, Rowland's Preliminary Table, Moore's Multiplet Table and our Table of U Cyg. ${ }^{7}$ In the spectral region which is common to all five stars, nearly 161 lines have been identified, of which 67 are of telluric origin. After

7) Y. Fujita: Ap. J., 116, 46 (1952). 
the identification was made, the lines which were sensibly free from blends were selected for the purpose of our discussion.

If we plot the estimated intensity against the lower excitation potential of each line, it is shown that the gradient of the mean

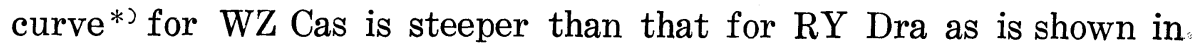
Fig. 1.

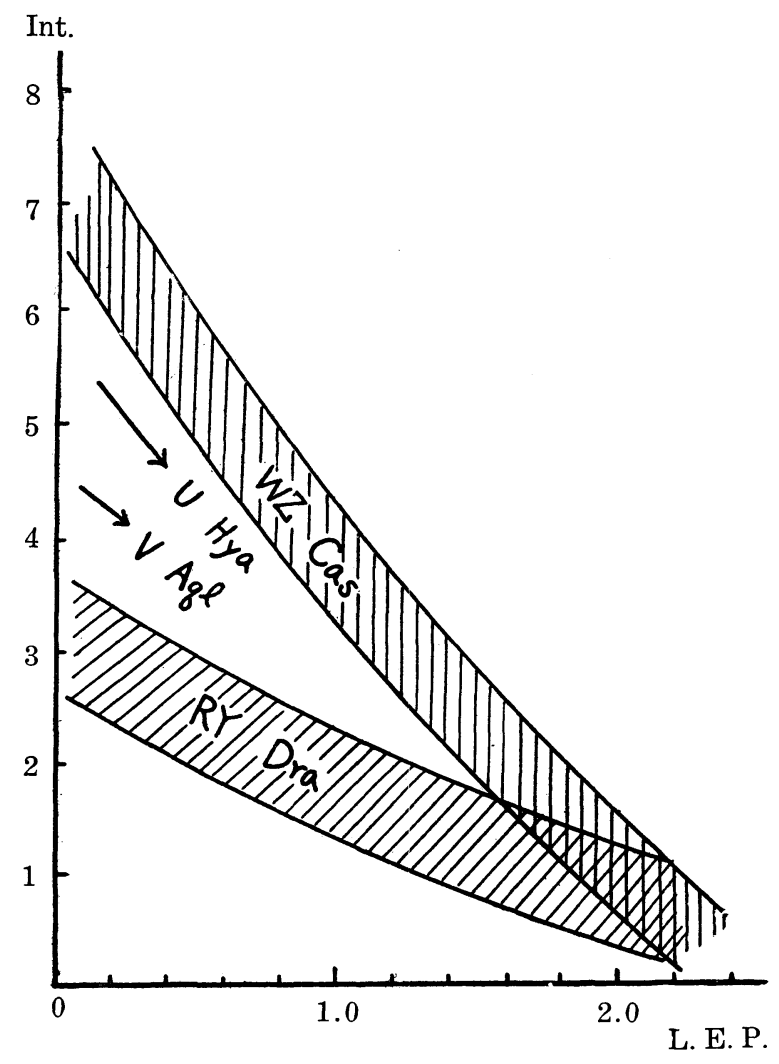

Fig. 1

Although the same curve for three other stars is not drawn in Fig. 1 , it can be seen that these gradients take the intermediate values between the former two. This means that the gradient is continuously getting steeper in the following isequence: C4-C6-C7-C9. This general tendency shows that the Keenan-Morgan classification is quite reasonable.

One of the remarkable features in the spectra of these five stars is the $\mathrm{K} 7699$ line. The microphotometric tracings from three of these are shown in Figs. 2-4.

Though it is very difficult to estimate the accurate value of the $\mathrm{K}$ intensity in each star, it is possible to give a general idea

*) From the intensity corrected by considering the neighbouring telluric lines of $\mathrm{O}_{2}$. 


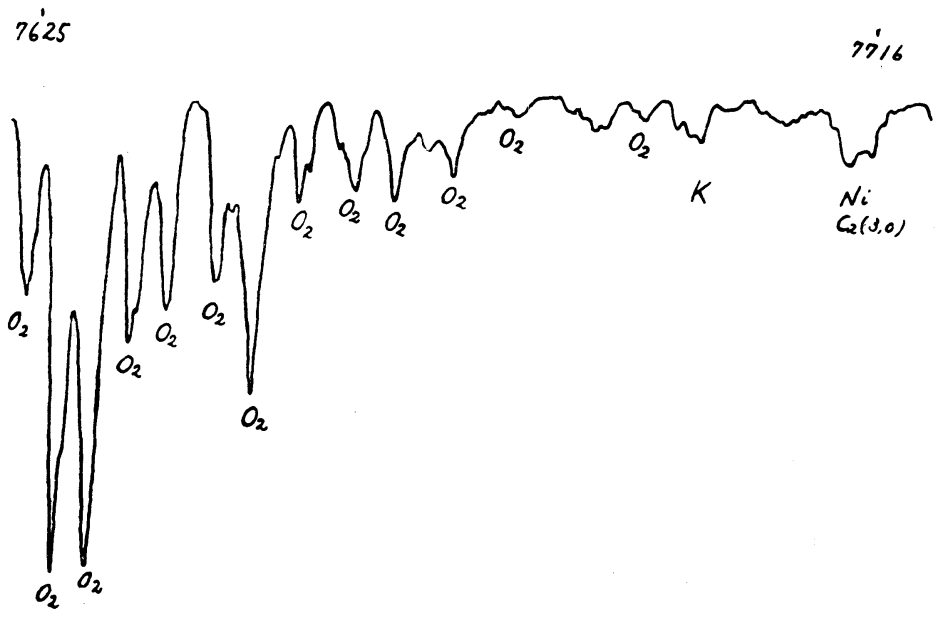

Fig. 2. RY Dra

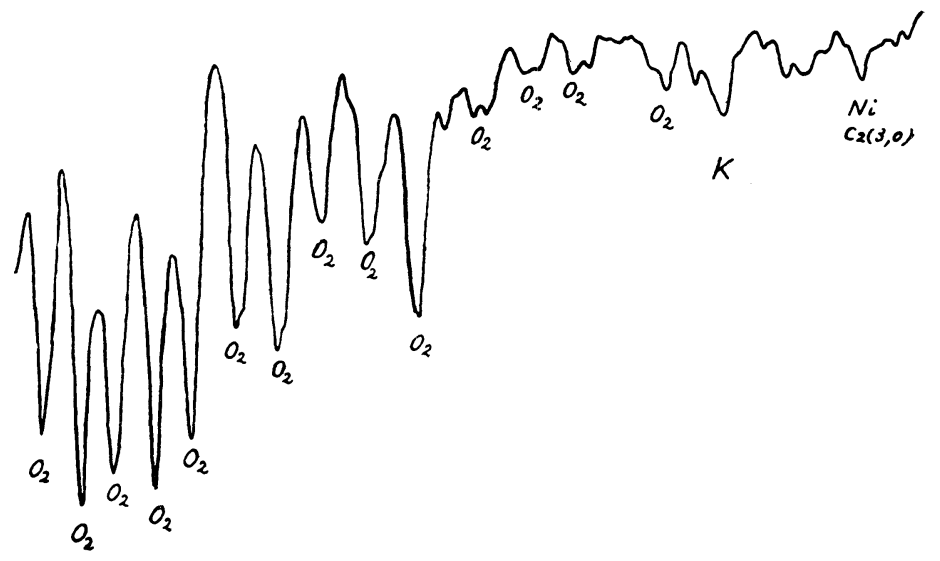

Fig. 3. U Cyg

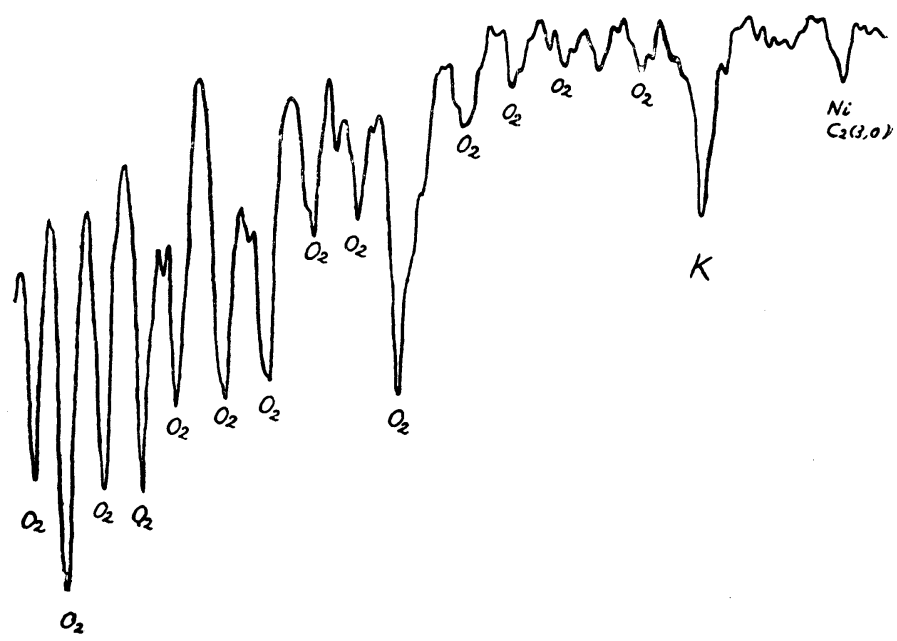

Fig. 4. WZ Cas 
of it, when we take the intensity of the telluric oxygen bands surrounding the line into consideration. The results show WZ Cas has the highest and RY Dra the lowest $K$ value.

\begin{tabular}{c|c|c|c|c|c}
\hline & WZ $\operatorname{Cas}\left(\mathrm{C}_{1}\right)$ & $\mathrm{U} \mathrm{Cyg}\left(\mathrm{C}_{2}-\mathrm{C}_{2}\right)$ & $\mathrm{U} \mathrm{Hya}\left(\mathrm{C}_{3}\right)$ & $\mathrm{V} \mathrm{Aql}\left(\mathrm{C}_{4}\right)$ & $\mathrm{RY} \operatorname{Dra}\left(\mathrm{C}_{4}\right)$ \\
\hline $\begin{array}{c}\text { Intensity of } \\
\text { K } 7699 \\
\text { (arbitrary } \\
\text { scale) }\end{array}$ & 6 & 4 & 3 & 2 & 2 \\
\hline
\end{tabular}

In the stellar atmosphere which is composed of $n$ kinds of elements, the number of neutral and ionized atoms per volume $N_{0}$ and $N_{1}$ can be written in the following equations: ${ }^{8)}$

$$
\begin{aligned}
N_{i}^{(i)}=\frac{\varepsilon_{i} P}{g \bar{m}} & -\frac{\varepsilon_{i}}{g \bar{m}} K_{i} \ln \frac{P+K_{i}}{K_{i}}+\frac{\varepsilon_{i}}{g \bar{m}} \frac{P^{2}}{P+K_{i}}\left[\sum \frac{\varepsilon_{i} K_{i}}{P+K_{i}}\right]^{-1} \\
& -\frac{\varepsilon_{i}}{g \bar{m}} \int_{0}^{P} \frac{K_{i} P}{\left(P+K_{i}\right)^{2}}\left[\sum \frac{\varepsilon_{i} K_{i}}{P+K_{i}}\right]^{-1} d P, \\
N_{1}^{(i)}= & \frac{\varepsilon_{i} K_{i}}{g \bar{m}} \ln \frac{P+K_{i}}{K_{i}}+\frac{\varepsilon_{i} K_{i}}{g \bar{m}} \frac{P}{P+K_{i}}\left[\sum \frac{\varepsilon_{i} K_{i}}{P+K_{i}}\right]^{-1} \\
& +\frac{\varepsilon_{i}}{g \bar{m}} \int_{0}^{P} \frac{K_{i} P}{\left(P+K_{i}\right)^{2}}\left[\sum \frac{\varepsilon_{i} K_{i}}{P+K_{i}}\right]^{-1} d P
\end{aligned}
$$

and

$$
\bar{m}=\sum_{i=1}^{n} \varepsilon_{i} m_{i}, \quad(i=1,2, \ldots, n)
$$

where $\varepsilon_{1}, \varepsilon_{2}, \ldots$ mean abundance of the elements; $m_{1}, m_{2}, \ldots$ atomic weight; $K_{1}, K_{2}, \ldots$ ionization function; $P$ electron pressure and $g$ surface gravity. $P$ and $g$ are easily calculated according to the luminosity types, as was shown in our paper. ${ }^{9}$. Avoiding the difficulty in the calculation of the integrals in (1) and (2), we can estimate the approximate values of $N_{i}^{(i)}$ and $N_{1}^{(i)}$ following the method described in our paper. ${ }^{10}$ In this way we calculated the number of atoms of $\mathrm{K}$ element between the temperature range of $1500^{\circ}$ to $5000^{\circ}$ for two types of the luminosity class. It is shown that the number of $\mathrm{K}$ element becomes maximum around $1600^{\circ}$ in case of the giant stars and $2400^{\circ}$ in case of the dwarf stars. The Keenan-Morgan classification well fits to the temperature scale. C9 is the lowest and it increases with the decreasing suffix number of C. Thus it should be concluded WZ Cas is one of the coolest stars, the temperature being nearly $1600^{\circ}$.

I should like to express my hearty thanks to Drs. W. W. Morgan and B. Strömgren for having put at my disposal the McDonald spectrograms and the Yerkes microphotometer.

8) Y. Fujita: Jap. Journ. Astr. Geophys., 13, 141 (1936).

9) Y. Fujita: Ibid., 17, 17 (1939).

10) Y. Fujita: Ibid., 13, 141 (1936). 